\title{
Epidemiologie und Prävention des Hautmelanoms in der Schweiz: Update 2010
}

\author{
Epidemiology and Prevention of Melanoma in Switzerland: 2010 Update
}

Autoren

Institute
J.-L. Bulliard ${ }^{1}$, R. G. Panizzon², F. Levi ${ }^{1,3}$

${ }^{1}$ Unité d'épidémiologie du cancer, Institut de médecine sociale et préventive, Centre Hospitalier Universitaire Vaudois et Université de Lausanne, Schweiz

2 Service de Dermatologie et Vénéréologie, Centre Hospitalier Universitaire Vaudois, Lausanne, Schweiz

${ }^{3}$ Registre Vaudois et Neuchâtelois des Tumeurs, Centre Hospitalier Universitaire Vaudois et Université de Lausanne, Schweiz

\section{Bibliografie}

DOI http://dx.doi.org/

10.1055/s-0030-1255817

Online-Publikation: 8. 10. 2010

Akt Dermatol 2010; 36:

408-413 ๑ Georg Thieme

Verlag KG Stuttgart · New York ISSN 0340-2541

\section{Korrespondenzadresse} PD Dr. MER Jean-Luc Bulliard Unité d'épidémiologie du cancer

Institut de médecine sociale et préventive

Centre Hospitalier

Universitaire Vaudois et Université de Lausanne Route de la Corniche 2 Bâtiment Biopôle 1 1066 Epalinges Schweiz

Jean-Luc.Bulliard@chuv.ch

\section{Zusammenfassung \\ $\nabla$}

Die Inzidenz des malignen Melanoms steigt seit über 50 Jahren bei der weißen Bevölkerung stark an. Die Schweiz ist mit ungefähr 1900 neu diagnostizierten Fällen pro Jahr das am stärksten betroffene Land Europas (16/100000 Welt-standardisierte Rate). In letzter Zeit sind regionale Unterschiede mit höherer Inzidenz in den Westschweizer Kantonen festzustellen. Änderungen in Wissen und Verhalten der Schweizer Bevölkerung gegenüber dem Schutz vor Sonnenexposition beste-

\section{Einleitung}

Das Melanom: ein Public-Health-Problem

Die Inzidenz des Melanoms, des schwersten Krebses an der Haut, steigt seit über einem halben Jahrhundert an [1]. Am stärksten sind hellhäutige Menschen in den Industrieländern betroffen. In diesen Ländern ist das Melanom im Allgemeinen die Krebsart, die am meisten zunimmt; seine Häufigkeit verdoppelt sich hier alle 15 bis 20 Jahre.

Die Inzidenz variiert um einen Faktor von mehr als 100 zwischen Australien mit der höchsten Inzidenz (55 Männer und 40 Frauen auf 100000 Einwohner pro Jahr) und Afrika sowie Teilen Asiens [2]. Jährlich werden weltweit etwa 200000 Fälle neu diagnostiziert, davon etwa 86000 in Europa [3]. In Europa ist das Melanom bei der Frau häufiger als beim Mann, in Nordamerika und Ozeanien dagegen wird das Gegenteil beobachtet [2] ( $\bullet$ Abb. 1).

Da es für disseminierte Melanome noch keine wirksame Therapie gibt und die Behandlung vor allem palliativ bleiben muss, ist die Prognose quoad vitam bei erst im fortgeschrittenen Stadium diagnostizierten Fällen ungünstig. Auf der anderen Seite bringt der chirurgische Eingriff im Frühstadium in vielen Fällen Heilung. Der beste prädiktive Faktor für die Überlebensdauer ist die hen noch zu wenig lange und sind zu bescheiden, als dass sie schon einen Einfluss auf die Inzidenz hätten haben können. Dank der seit 20 Jahren betriebenen Früherfassung sind Überlebensrate und Anteil an dünnen Melanomen gestiegen, allerdings bei gleichbleibender Inzidenz dicker Läsionen. Die Mortalität aufgrund des malignen Melanoms ist neuerdings rückläufig, vor allem bei den Frauen. Werden die gegenwärtigen Präventionsbemühungen weitergeführt, dürften sich bald noch mehr Erfolge zeigen.

Dicke der Läsion (nach Breslow). Europäische Angaben über Patienten verzeichnen bei dünnen Läsionen von $<1 \mathrm{~mm}$ 5-Jahres-Überlebensraten von $95 \%$, bei dicken Läsionen von $>4 \mathrm{~mm}$ dagegen lediglich um die $42 \%$ bis $65 \%[4,5]$.

Das Melanom verursacht 46000 Todesfälle im Jahr, davon 21000 in Europa [3]. Zwar ist die Mortalität in den Ländern mit hoher Inzidenz höher, jedoch sind die entsprechenden Unterschiede zwischen den Ländern geringer als diejenigen der Inzidenz und tendenziell weiter rückläufig $[1,6]$. So ist die Mortalität an Melanom in Nordund Westeuropa etwa um 50\% höher als in Ostund Südeuropa, während die Inzidenz hier 2- bis 3-mal tiefer liegt als im Westen und Norden $(\bullet$ Abb. 1). Dies dürfte, zumindest teilweise, auf eine frühe Erfassung und in gewissem Ausmaß vielleicht auch auf ein prompteres Vorgehen bei Diagnose und Behandlung in Ländern mit hoher Inzidenz zurückzuführen sein [1] ( $\bullet$ Abb. 2).

\section{Die Situation in der Schweiz}

Die Schweiz ist durch das Melanom besonders stark betroffen; in den neuesten weltweiten Statistiken sind die Inzidenzraten in der Westschweiz die höchsten Europas [2]. Extrapoliert man die routinemäßig durch die kantonalen Krebsregister erhobenen Daten, kommt man für 


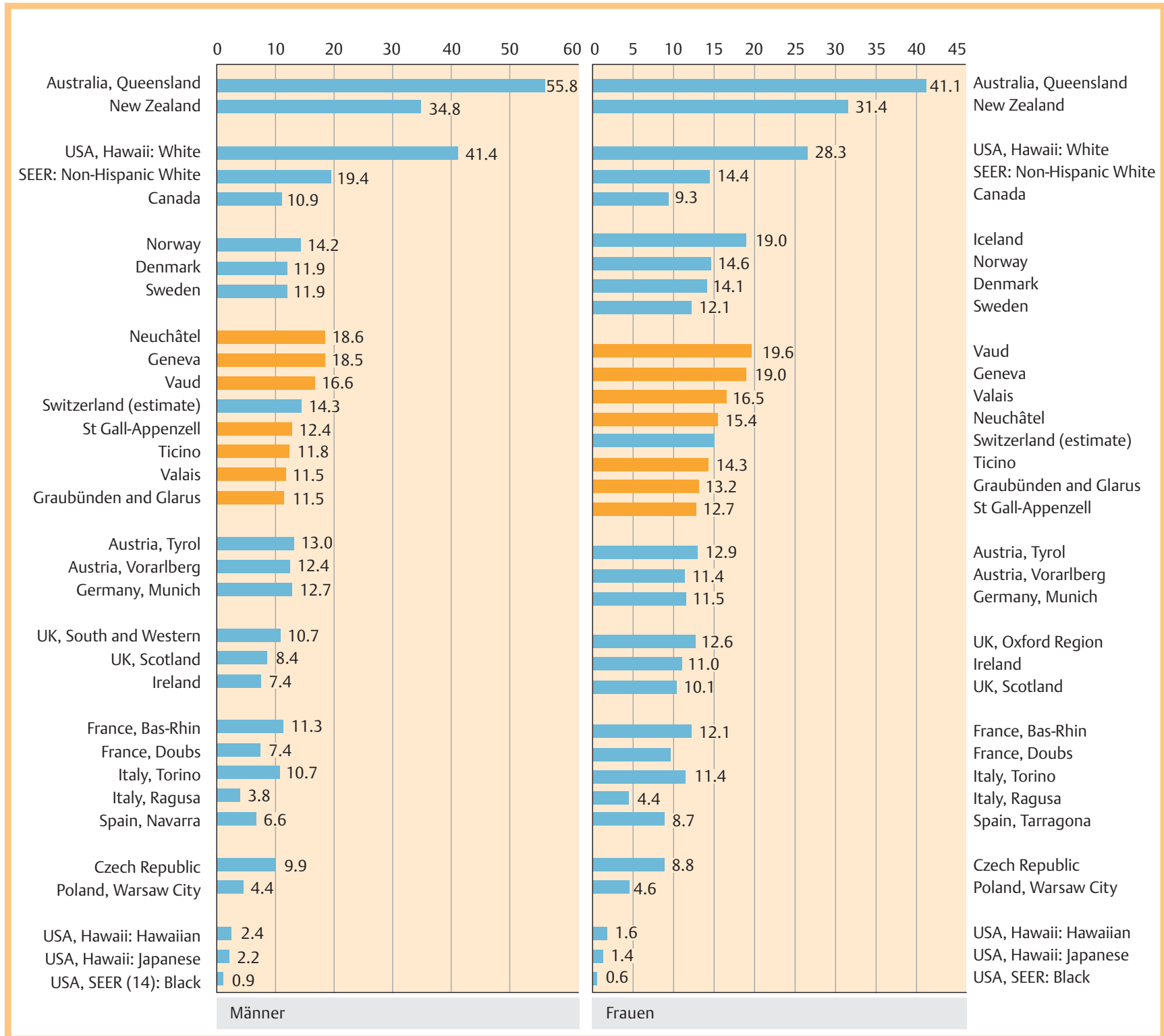

Abb. 1 Altersstandardisierte Inzidenz (Weltbevölkerung) von Melanom nach Geschlecht in ausgewählten Ländern und Regionen 1998 bis 2002 [2, 7].

die gesamte Schweiz auf jährlich rund 1900 neu diagnostizierte Fälle von Melanom, davon die Hälfte vor Alter 60 [7]. Altersstandardisiert (Weltbevölkerung) beträgt die Rate 16 Fälle auf 100000 Einwohner pro Jahr ( $\mathbf{A b b . 1}$ ). Damit ist das Melanom der vierthäufigste Krebs sowohl bei Männern als auch bei Frauen und ist für $5 \%$ der bösartigen Tumoren in der Schweiz verantwortlich [7]. Bei Erwachsenen unter 40 Jahren ist das Melanom in der Schweiz sogar die zweithäufigste Krebserkrankung. Da die Erkrankung relativ früh im Leben auftritt, bedeutet im Durchschnitt jeder Todesfall 15 verlorene potenzielle Lebensjahre; das bedeutet insgesamt einen Verlust an potenziellen Lebensjahren, der demjenigen durch Leberzirrhose oder dem Doppelten durch Diabetes entspricht.

In der Schweiz nimmt das Melanom um etwa 3\% pro Jahr zu (๑ Abb. 3).

Diese Entwicklung hält nun schon seit mehreren Jahrzehnten an. Die bei der Schweizer Bevölkerung zwischen 20 und 49 Jahren beobachtete Zunahme von 2,7\% pro Jahr lässt leider kaum auf eine baldige Änderung dieses Trends schließen. Vor allem die dünnen Melanome mit einer guten Heilungschance haben zugenommen, was auf eine verbesserte Sensibilisierung und immer frühere $\mathrm{Er}$ fassung dieser Tumoren zurückzuführen ist. Demgegenüber hat sich die Inzidenzrate der dicken Melanome mit deutlich schlechterer Prognose in den letzten 15 Jahren kaum verändert.

Am deutlichsten haben sowohl in der Schweiz als auch im Ausland die Tumoren an den intermittierend dem Sonnenlicht ausgesetzten Lokalisationen (Rumpf und Arme) und - was den histologischen Typ anbelangt - das oberflächlich spreitende Melanom zugenommen [8]. Bemerkenswert ist der relativ hohe Anteil von Melanomen am Kopf, Hals und Nacken bei der Schweizer Bevölkerung. Diese Besonderheit, die man übrigens auch bei der Tiroler Bevölkerung antrifft, könnte auf eine Exposition in höheren Lagen mit entsprechend starker Sonneneinstrahlung beim Wandern, Skisport, Alpinismus durch das ganze Jahr hindurch zurückzuführen sein [9].

280 Schweizer Einwohner sterben jährlich an Melanomen. Die Mortalität ist bei Frauen geringer, da sie im Allgemeinen ihre Haut regelmäßiger inspizieren und bei suspekten Läsionen eher den Arzt aufsuchen. Die Mortalität ging bei Frauen in der Schweiz zu Beginn der 1990er-Jahre zurück und liegt nun seit 10 Jahren 


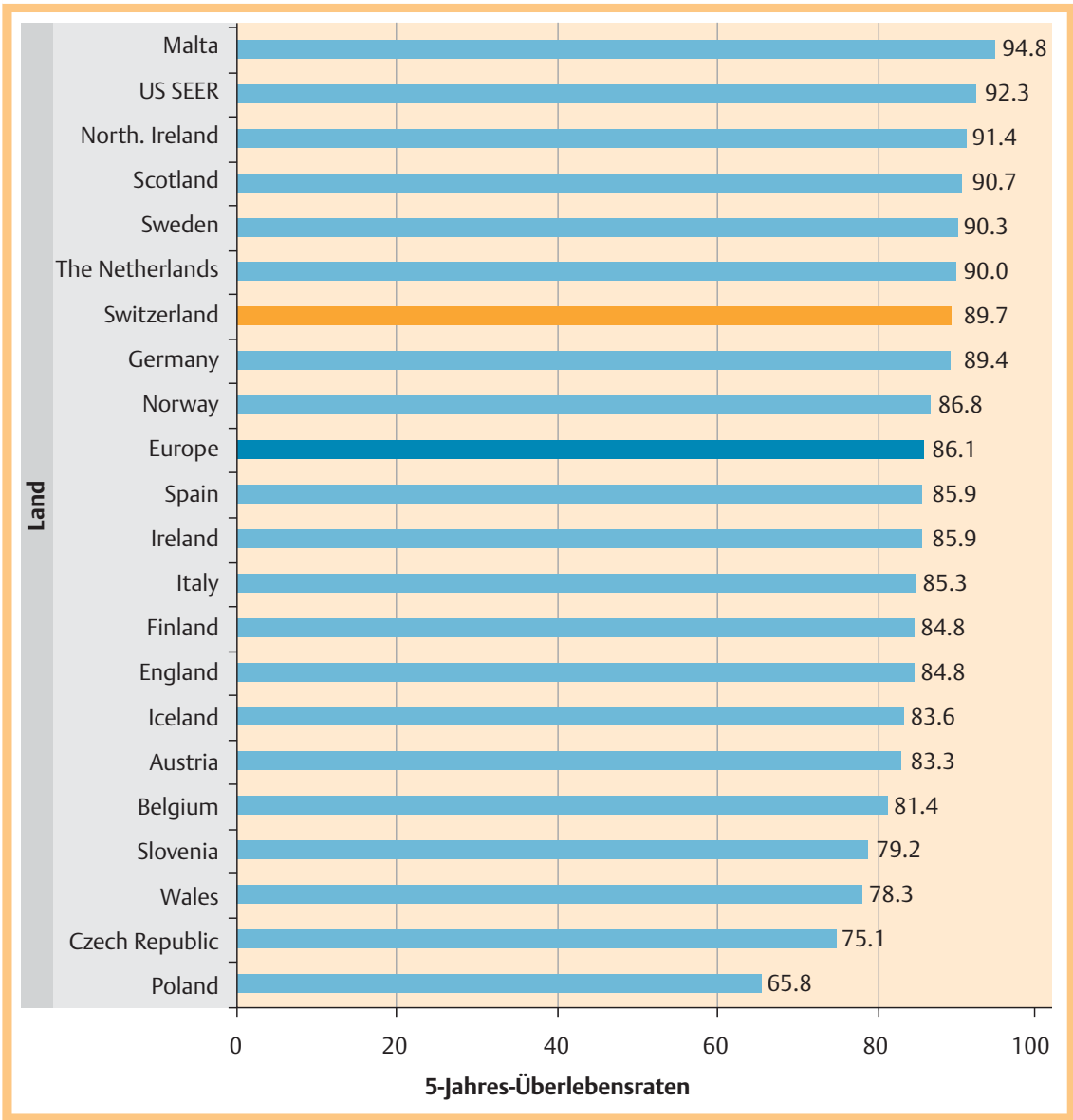

Abb. 2 5-Jahres-Überlebensrate bei Patienten mit einem zwischen 2000 und 2002 diagnostizierten Melanom in Europa und den USA (nach EUROCARE-4 [14], adaptiert. Die Schätzungen für die Schweiz beruhen auf den Daten der kantonalen Krebsregister beider Basel, Genf, Graubünden/ Glarus, St. Gallen/Appenzell, Wallis, Tessin und Zürich).

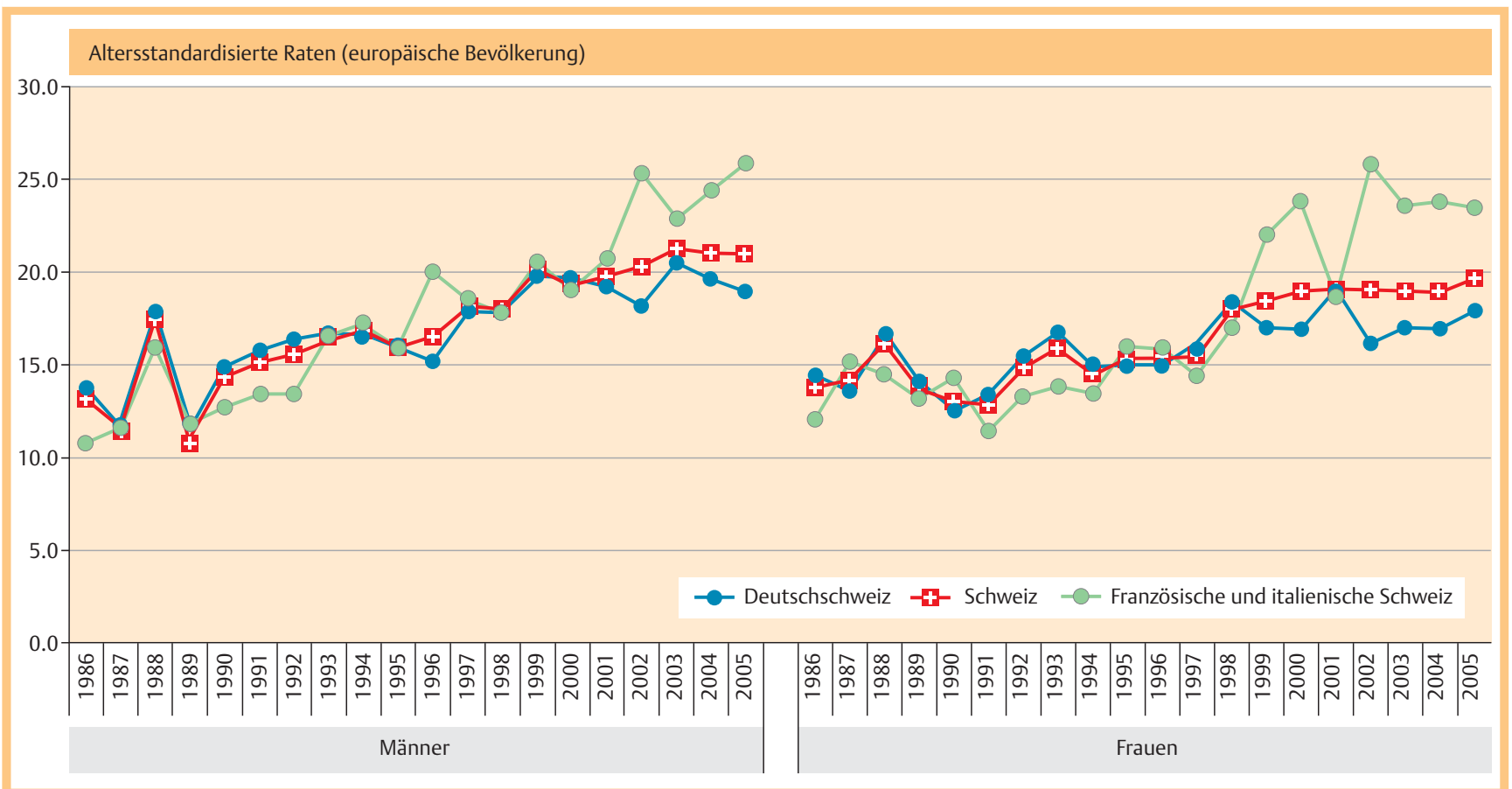

Abb. 3 Zeitliche Entwicklung der Inzidenz von Melanom nach Geschlecht und Sprachregion. Schweiz, 1986-2005 (Quelle: National Institute for Cancer Epidemiology and Registration). 


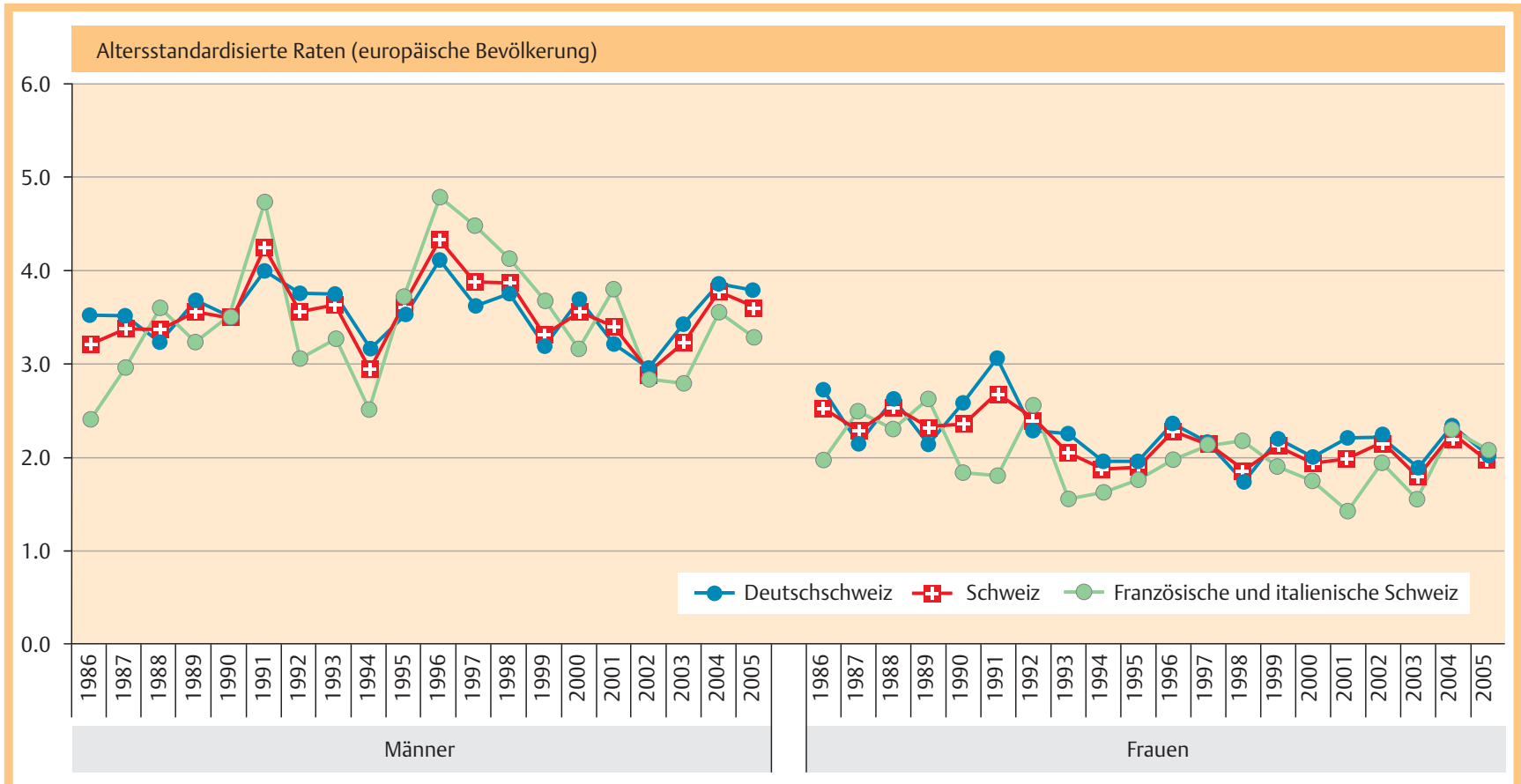

Abb. 4 Zeitliche Entwicklung der Mortalität von Melanom nach Geschlecht und Sprachregion. Schweiz, 1986-2005 (Quelle: Bundesamt für Statistik und National Institute for Cancer Epidemiology and Registration).

gleichbleibend bei etwa 2 Todesfällen auf 100000 Einwohner (๑ Abb. 4).

Bei den Männern fiel die Abnahme deutlicher aus, obschon sie erst später, um 1998, begonnen hatte. International gesehen wurde in den Ländern, die als erste Präventionskampagnen auf die Beine gestellt hatten (wie z.B. Australien bereits in den 1960er-Jahren), auch zuerst ein Rückgang der Melanommortalität gemeldet. Ähnlich verläuft die Entwicklung in mehreren anderen europäischen Ländern, wo die Mortalität auch bei jungen Altersgruppen rückläufig ist [6]. Überall wurde dieser Rückgang, gleich wie in der Schweiz, zuerst bei den Frauen beobachtet. In den letzten Jahren war der Anstieg der Inzidenz in der französischsprachigen Schweiz deutlicher als in der Deutschschweiz [7]. So betrug die Zunahme der Inzidenzrate in der Periode 1994 bis 2005 in der französischen und italienischen Schweiz 4\% pro Jahr, in der Deutschschweiz dagegen bloß 2\% ( Abb. 3). Dieser regionale Unterschied ist bei den Frauen mit 6,5\% bzw. 1,4\% jährlichem Wachstum noch deutlicher. Weitere Analysen sind nötig, um dieses Phänomen besser verstehen zu können, denn bei der Mortalität haben die Werte umgekehrt bei den Männern aus der französischsprachigen Schweiz mit 2,6\% pro Jahr stärker abgenommen als bei den Deutschschweizern (0,5\% pro Jahr), was im Widerspruch steht zu den neueren Tendenzen bei den Frauen in den jeweiligen Sprachregionen ( $\bullet$ Abb.4).

\section{Prävention der Hautkrebse \\ $\nabla$}

\section{Entscheidend ist die Sonnenexposition}

Die von der Sonne emittierten und von der Ozonschicht nicht zurückgehaltenen UV-Strahlen sind für etwa $80 \%$ der Krebserkrankungen an der Haut verantwortlich. Dieses Karzinogen spielt sowohl bei der Initiation wie bei der Promotion von Hautkrebs eine Rolle. Auch wenn unsere Kenntnisse über die Mechanismen der Photokarzinogenese durch UV-Licht lückenhaft sind, ist doch klar, dass das individuelle Verhalten gegenüber Sonnenlicht entscheidend bestimmt, welche Strahlendosis das Individuum erhält. Die Familienanamnese sowie konstitutionelle Faktoren (Hauttyp, Augen- und Haarfarbe, Anzahl und Art von Naevi, genetische Prädisposition) sind weitere Risikofaktoren, die jedoch im Unterschied zur Exposition zu UV-Strahlen durch das Individuum nicht beeinflusst werden können.

Der höhere Lebensstandard (Reduktion der Arbeitszeit, erleichterter Zugang zu Ländern mit hoher Sonneneinstrahlung), vermehrte Freizeitbeschäftigung im Freien sowie eine Kleidermode, die zunehmend unbedeckte Haut zulässt, haben dazu geführt, dass die Haut vermehrt dem Sonnenlicht ausgesetzt ist. Anfang des 20. Jahrhunderts war ein gebräunter Teint ein Zeichen für harte körperliche Arbeit einer niedrigen Bevölkerungsschicht; heute dagegen bedeutet gebräunte Haut ein Privileg, das eine vor allem wohlhabende Gesellschaft und die Jugendlichen auszeichnet, die sich dem Sonnenlicht oft intermittierend, dafür intensiv aussetzen. Diese Art der Exposition ist besonders schädlich für die Haut, und sie dürfte zum Teil erklären, weshalb das Hautmelanom in wohlhabenden Ländern und privilegierten sozialen Schichten so häufig geworden ist. Chronische Exposition auf der anderen Seite spielt bei der Entstehung der übrigen Typen von Hautkrebs, in erster Linie des Spinalioms, eine wichtige Rolle.

\section{Arten von Prävention, Erfolgskriterien}

Man unterscheidet die primäre Prävention, deren Ziel in der Information über Risikofaktoren und der Möglichkeiten, sich dagegen zu schützen, besteht, von der sekundären Prävention, die eine möglichst frühzeitige Erfassung der Läsionen zum Ziel hat. Theoretisch führt eine erfolgreiche primäre Prävention mit der Zeit zu einer Abnahme der Inzidenz (und im Gefolge davon der Mortalität), während eine erfolgreiche Früherfassung sich in einer Verbesserung der Überlebensdauer und Senkung der Mortalität an der entsprechenden Krebserkrankung niederschlägt. 
Praktisch erlaubt lediglich der zeitliche Verlauf der Inzidenz an dicken Melanomen abzuschätzen, wieweit Informationskampagnen (Primärprävention) effektiv einen Einfluss auf die Krankheitshäufigkeit haben. Die Inzidenz der dünnen Melanome, die am meisten zur Gesamtinzidenz bei dieser Krebsart beitragen, steigt unter dem Einfluss der Früherfassungsmaßnahmen an [8]. Es kann daher irreführend sein, sich bei der Beurteilung von Präventionskampagnen lediglich auf einen abnehmenden prozentualen Anteil dicker Melanome oder die durchschnittliche (mediane) Dicke der Läsion bei der Diagnosestellung zu stützen. Darüber hinaus wird es bei der Erfassung immer dünnerer Läsionen auch zunehmend schwieriger, zu entscheiden, welche Läsionen exzidiert werden müssen und welche für den Patienten kaum oder gar keine Gefahr darstellen. Ohne zuverlässige prognostische Kriterien für den Entscheid zur Operation würden immer mehr zwar maligne Läsionen diagnostiziert, bei denen aber keinerlei Metastasierungsgefahr besteht [10]. Die Zunahme der Inzidenz an dünnen und In-situ-Melanomen ohne gleichzeitige Abnahme der dicken Läsionen spricht ebenfalls für diese Hypothese. Zwischen der UV-Exposition und dem Erscheinen des Hautkrebses können oft mehrere Jahrzehnte vergehen. Dies macht es für das Publikum schwierig, die Verbindung zwischen gegenwärtigem Verhalten und zukünftigem Risiko richtig wahrzunehmen. Trotzdem gibt es verschiedene epidemiologische Indikatoren, die eine günstige Entwicklung erwarten lassen. So steigt die Inzidenz bei den jungen Altersgruppen in den Ländern mit wirksamen Präventionsprogrammen seit vielen Jahren nicht mehr an. Den Erfolg der Früherfassung des Melanoms (sekundäre Prävention) misst man eher aufgrund der Mortalität denn der Überlebensdauer. Diese ist ein unzuverlässiger Indikator; dank der Früherfassung wird nämlich auch der Zeitpunkt der Diagnosestellung vorverlegt, wodurch sich die Zeit zwischen Diagnosestellung und Tod verlängert, was die Überlebenszeit unabhängig davon, ob eine echte Verbesserung vorliegt oder nicht, scheinbar verbessert (lead-time bias).

\section{Präventionsmaßnahmen in der Schweiz}

Die Schweiz gehört zu den ersten Ländern in Europa, die Informationskampagnen über die Gefahren übertriebener Sonnenexposition und Möglichkeiten, sich zu schützen, auf die Beine gestellt haben [11]. Begonnen wurde mit diesen Kampagnen vor 20 Jahren, die Programme umfassten sowohl primäre wie sekundäre Prävention und hatten verschiedene Bevölkerungsgruppen im Visier. Seit 2000 werden diese nationalen Kampagnen unter der Ägide der Schweizer Krebsliga unter Mitwirkung der Schweizerischen Gesellschaft für Dermatologie und Venerologie sowie des Bundesamts für Gesundheit durch die Fachkommission Hautkrebs der Schweizer Krebsliga koordiniert und evaluiert. So konnte zwischen 2000 und 2005 jeder Schweizer auf Wunsch im Rahmen der jährlichen „Sonnenmobil“-Kampagnen verdächtige Läsionen gratis untersuchen lassen, sich über seinen Hauttyp informieren und sein Hautkrebsrisiko selbst evaluieren. Auch eine Gratisuntersuchung durch einen Dermatologen wurde in dem „Sonnenmobil“, das das ganze Land durchreiste, angeboten $[11,12]$.

Seit 2006 organisiert die Schweiz im Monat Mai gemeinsam mit einigen anderen europäischen Ländern einen nationalen Hautkrebstag („Euro-Melanoma Day“). Die beteiligten Arztpraxen und Spitäler bieten Risikopersonen klinische Untersuchungen an. Da die Aktion vielerorts gleichzeitig stattfindet und zeitlich flexibel ist, können mehr Personen erfasst werden, und die Kampagne wirkt auch gezielter (hauptsächlich sekundäre Pro- phylaxe) als „Sonnenmobil“-Aktionen. Durch eine Nacherfassung der verdächtigen Läsionen bei den Krebsregistern kann die Qualität der durchgeführten Früherfassungsuntersuchungen abgeschätzt werden [13].

Umfragen in der Schweizer Bevölkerung zeigen eine langsame Verbesserung der Kenntnisse über die schädlichen Auswirkungen der UV-Strahlung und über Möglichkeiten, sich davor zu schützen. Es sind bescheidene, aber doch ermutigende Änderungen in der Einstellung und dem Verhalten der Schweizer Bevölkerung gegenüber Sonnenlicht zu beobachten. Allerdings wird eine sonnengebräunte Haut von der Gesellschaft nach wie vor als Zeichen guter Gesundheit gewertet; es bleibt also noch viel Erziehungsarbeit für die primäre Prävention zu tun.

Da keine wesentlichen therapeutischen Fortschritte zu verzeichnen sind, darf der aktuelle Rückgang der Mortalität als Erfolg der Früherfassung gewertet werden. Tatsächlich kann es die Heilungschancen erheblich verbessern, wenn bei Auftreten einer verdächtigen Läsion (Erscheinen eines neuen pigmentierten Flecks, eines Leberflecks mit veränderter Form oder Farbe, eines bestehenden Flecks, der zu jucken, zu bluten oder zu schmerzen beginnt) sofort ein Arzt aufgesucht wird. Wie viel allerdings die Kampagnen zu diesem Erfolg beigetragen haben, bleibt unbekannt.

Neben diesen im Rahmen der öffentlichen Präventionsarbeit verbreiteten Botschaften und Empfehlungen ist auch die Rolle des Hausarztes von eminenter Bedeutung: Die Patienten für die Gefahren der Bräunung an der Sonne sensibilisieren; erklären, warum Sitzungen im Solarium nicht zu empfehlen sind, nicht einmal, um die Haut für die nächsten Ferien „Vorzubereiten“; die Möglichkeiten und Grenzen von Sonnenschutzmitteln - die ja das Erythem zu verzögern, aber nicht gänzlich zu verhindern vermögen, weil sie einen Teil der UV-Strahlen durchlassen - klarzustellen; die Gefahren aufzuzeigen, wenn man Sonnenschutzmittel dazu missbraucht, nur desto länger an der Sonne zu liegen; die Patienten im Hinblick auf die Versorgung mit Vitamin D zu beruhigen, d.h. klarzustellen, dass eine mäßige, mit den Empfehlungen zum Schutz vor übertriebener Strahlung durchaus vereinbare Sonnenexposition auch in dieser Hinsicht vollauf genügt. Die Untersuchung verdächtiger Läsionen in immer früheren Stadien auf der anderen Seite sollte dem Spezialisten vorbehalten bleiben, denn deren Beurteilung ist heikel und von großer Tragweite.

\section{Schlussfolgerungen \\ $\nabla$}

Das Melanom stellt in der Schweiz ein wichtiges Public-HealthProblem dar. Wir beobachten hier eine der höchsten Inzidenzraten in ganz Europa. Heute können wir in der Schweiz die ersten Erfolge der im Laufe der letzten 20 Jahre auf verschiedenen Wegen an verschiedene Zielgruppen herangetragenen Informationskampagnen zur primären Prävention beobachten. Es lohnt sich also, mit diesen Bemühungen fortzufahren und diese so gut als möglich zu dokumentieren. Die Früherfassung - vor allem bei Risikopersonen - hat zu einer deutlichen Verbesserung der Überlebenszeit geführt, und die Mortalität an Melanom sollte in der Schweiz weiterhin zurückgehen. Wir empfehlen eine vertiefte epidemiologische Analyse der soziodemografischen und klinischen Faktoren, die Inzidenz und Mortalität an Melanom beeinflussen, sowie eine eingehende Evaluation der Präventionskampagnen. 


\section{Abstract}

\section{Epidemiology and Prevention of Melanoma in Switzerland: 2010 Update}

The incidence of cutaneous malignant melanoma has steadily increased in Caucasian populations over the last decades. With around 1900 new cases each year, Switzerland has one of the highest melanoma rates in Europe (16/100000 world-standardised rate).

Regional differences are emerging within Switzerland, with a higher incidence in the western (French-speaking) region. Observed changes in sun protection attitudes and knowledge in the Swiss population have yet no impact on the incidence trend. Early detection, carried out since the mid 1980s in Switzerland, has led to a substantial increase in survival and rates of thin melanoma, without material change in rates of thick melanoma. Mortality from melanoma has recently decreased, earlier in women than men. The efficacy of prevention campaigns should soon become more blatant if current efforts persist.

\section{Literatur}

1 de Vries E, Bray FI, Coebergh JW, Parkin DM. Changing epidemiology of malignant cutaneous melanoma in Europe 1953-1997: rising trends in incidence and mortality but recent stabilizations in western Europe and decreases in Scandinavia. Int J Cancer 2003; 107: 119- 126

2 Curado MP, Edwards B, Shin HR et al. Cancer Incidence in Five Continents. IARC Sci. Publ. No. 160, Vol. IX. Lyon, France: International Agency for Research on Cancer, 2007

3 Ferlay J, Shin HR, Bray F et al. GLOBOCAN 2008: Cancer Incidence and Mortality Worldwide: IARC CancerBase No. 10 [Internet]. Lyon:
France: International Agency for Research on Cancer, 2010; Available from: http://globocan.iarc.fr; Accessed on 1/9/2010

4 de Vries E, Houterman S, Janssen-Heijnen ML et al. Up-to-date survival estimates and historical trends of cutaneous malignant melanoma in the south-east of The Netherlands. Ann Oncol 2007; 18: 1110-1116

5 Downing A, Newton-Bishop JA, Forman D. Recent trends in cutaneous malignant melanoma in the Yorkshire region of England; incidence, mortality and survival in relation to stage of disease, 1993-2003. Br J Cancer 2006; 95: 91 - 95

6 La Vecchia C, Bosetti C, Lucchini F et al. Cancer mortality in Europe, 2000-2004, and an overview of trends since 1975. Ann Oncol 2009; 21: $1323-1360$

7 National Institute for Cancer Epidemiology and Registration and Swiss Federal Office of Statistics. Gesundheit der Bevölkerung - Daten, Indikatoren Hautmelanom 2003-2007. Zurich and Neuchâtel: Statistik Schweiz, 2010; Available from http://www.bfs.admin.ch, Accessed on $1 / 9 / 2010$

8 Levi F, Te VC, Randimbison L, La Vecchia C. Trends in incidence of various morphologies of malignant melanoma in Vaud and Neuchatel, Switzerland. Melanoma Res 2005; 15: 73-75

9 Bulliard J-L, De Weck D, Fisch T et al. Detailed site distribution of melanoma and sunlight exposure: aetiological patterns from a Swiss series. Ann Oncol 2007; 18: 789-794

10 Burton RC, Armstrong BK. Recent incidence trends imply a nonmetastasizing form of invasive melanoma. Melanoma Res 1994; 4: 107 - 113

11 Bulliard J-L, Raymond L, Levi F et al. Prevention of cutaneous melanoma: an epidemiological evaluation of the Swiss campaign. Rev Epidémiol Santé Publique 1992; 40: 431 - 438

12 Bulliard J-L, Levi F, Panizzon RG. Campagne de prévention „Solmobile“ 2003 des cancers cutanés de la Ligue suisse contre le cancer: résultats et enjeux. Rev Med Suisse Romande 2004; 124: 237-240

13 Bulliard J-L, Maspoli M, Panizzon RG et al. Evaluation of the Euromelanoma skin cancer screening campaign: the Swiss experience. J Eur Acad Dermatol Venereol 2008; 22: 365-366

14 Verdecchia A, Francisci S, Brenner $\mathrm{H}$ et al. Recent cancer survival in Europe: a 2000-02 period analysis of EUROCARE-4 data. Lancet Oncol 2007: 8: 784-796 